\title{
The Effects of Obesity on Lymphatic Pain and Swelling in Breast Cancer Patients
}

\author{
Mei Rosemary Fu 1,*,+(D), Deborah Axelrod ${ }^{2,+}$, Amber Guth ${ }^{2}$, Melissa L. McTernan ${ }^{3}$, Jeanna M. Qiu 4 (D), \\ Zhuzhu Zhou ${ }^{3}$, Eunjung Ko ${ }^{5}$, Cherlie Magny-Normilus ${ }^{6}$, Joan Scagliola ${ }^{7}$ and Yao Wang ${ }^{8}$ \\ 1 School of Nursing-Camden, Rutgers, The State University of New Jersey, Camden, NJ 08102, USA \\ 2 Department of Surgery, School of Medicine, New York University, New York, NY 10016, USA; \\ Deborah.Axelrod@nyulangone.org (D.A.); Amber.Guth@nyulangone.org (A.G.) \\ 3 Boston College Research Services, Chestnut Hill, MA 02467, USA; mcternam@bc.edu (M.L.M.); \\ zhouzp@bc.edu (Z.Z.) \\ 4 Harvard Medical School, Boston, MA 02115, USA; jeanna_qiu@hms.harvard.edu \\ 5 The Ohio State University College of Nursing, Columbus, OH 43210, USA; ko.363@buckeyemail.osu.edu \\ 6 Boston College William F. Connell School of Nursing, Chestnut Hill, MA 02467, USA; \\ cherlie.magny-normilus@bc.edu \\ 7 NYU Laura and Isaac Perlmutter Cancer Center, New York, NY 10016, USA; Joan.Scagliola@nyulangone.org \\ 8 Tandon School of Enginereeng, Electrical and Computer Engeenerng and Biomedical Engineering, \\ New York Universuty, New York, NY 11202, USA; yaowang@nyu.edu \\ * Correspondence: mei.r.fu@rutgers.edu or mf67@nyu.edu \\ + Mei Rosemary Fu and Deborah Axelrod shared the first author.
}

Citation: Fu, M.R.; Axelrod, D.; Guth, A.; McTernan, M.L.; Qiu, J.M.; Zhou, Z.; Ko, E.; Magny-Normilus, C.; Scagliola, J.; Wang, Y. The Effects of Obesity on Lymphatic Pain and Swelling in Breast Cancer Patients. Biomedicines 2021, 9, 818. https:// doi.org/10.3390/biomedicines 9070818

Academic Editor: Vittoria D'Esposito

Received: 27 May 2021

Accepted: 6 July 2021

Published: 14 July 2021

Publisher's Note: MDPI stays neutral with regard to jurisdictional claims in published maps and institutional affiliations.

Copyright: (c) 2021 by the authors. Licensee MDPI, Basel, Switzerland. This article is an open access article distributed under the terms and conditions of the Creative Commons Attribution (CC BY) license (https:// creativecommons.org/licenses/by/ $4.0 /)$.
Abstract: Abstract: BackgroundLymphatic pain and swelling due to lymph fluid accumulation are the most common and debilitating long-term adverse effects of cancer treatment. This study aimed to quantify the effects of obesity on lymphatic pain, arm, and truncal swelling. Methods: A sample of 554 breast cancer patients were enrolled in the study. Body mass index (BMI), body fat percentage, and body fat mass were measured using a bioimpedance device. Obesity was defined as a BMI $\geq 30 \mathrm{~kg} / \mathrm{m}^{2}$. The Breast Cancer and Lymphedema Symptom Experience Index was used to measure lymphatic pain, arm, and truncal swelling. Multivariable logistic regression models were used to estimate the odds ratio (OR) with 95\% confidence interval (CI) to quantify the effects of obesity. Results: Controlling for clinical and demographic characteristics as well as body fat percentage, obesity had the greatest effects on lymphatic pain (OR 3.49, 95\% CI 1.87-6.50; $p<0.001$ ) and arm swelling (OR 3.98, 95\% CI 1.82-4.43; $p<0.001$ ). Conclusions: Obesity is a significant risk factor for lymphatic pain and arm swelling in breast cancer patients. Obesity, lymphatic pain, and swelling are inflammatory conditions. Future study should explore the inflammatory pathways and understand the molecular mechanisms to find a cure.

Keywords: obesity; lymphatic; pain; swelling; arm swelling; truncal swelling; lymphedema; breast cancer

\section{Introduction}

Obesity is the accumulation of body fat and an inflammatory condition [1-4]. The standard criterion to define obesity is a body mass index (BMI) $\geq 30 \mathrm{~kg} / \mathrm{m}^{2}$ [1,3]. Obesity not only increases the risk of diagnosis and poor prognosis of breast cancer [5-7] but also the risk of developing long-term and debilitating adverse effects of cancer treatment [8,9]. Lymphatic pain and swelling due to lymph fluid accumulation are the most common and debilitating long-term adverse effects of cancer treatment that negatively impact breast cancer patients' quality of life $[10,11]$. Over $90 \%$ of women treated for breast cancer have achieved five-year survival due to advances in cancer diagnosis and treatment $[12,13]$. Currently, more than 3.8 million women treated for breast cancer are living in the United States and more than 7.8 million worldwide [12,13]. Yet, there are more lost disabilityadjusted life years by women to breast cancer globally than any other types of cancer [12]. 
The long-term and debilitating adverse effects of cancer treatment, such as pain, swelling and lymphedema, may be the major causes for the most lost disability-adjusted life years.

Patient-centered health outcomes are defined by the Patient-Centered Outcomes Research Institute (PCORI) as health outcomes that people notice and care about, such as symptoms, function, quality of life, and survival [14]. Patient-centered outcomes reflect patients' experience in disease management and are critical indicators that help patients and healthcare providers to make ongoing value-based treatment and care decisions. Lymphatic pain refers to a variety of pain sensations (i.e., pain/aching/soreness) accompanied by swelling in the ipsilateral body or upper limb due to a compromised lymphatic system following cancer treatment [15]. Lymphedema is a chronic and incurable condition caused by an abnormal accumulation of lymph fluid in the ipsilateral body or upper limb after cancer treatment [16]. Swelling and lymphedema reflect an inflammatory pathological condition $[17,18]$. Swelling refers to patient-reported symptom, such as swelling in the ipsilateral upper limb (i.e., arm swelling) or upper body of breast or chest wall (i.e., truncal swelling), while lymphedema is often defined as an increased limb size or girth comparing affected (or lymphedematous) and unaffected limbs [19]. As the cardinal symptoms of early-stage lymphedema, lymphatic pain and swelling (i.e., arm swelling and truncal swelling) are symptoms that often precede changes in limb size or girth or a lymphedema diagnosis [18]. Without timely intervention, this early disease stage can progress into lymphedema that no surgical or medical interventions can cure [16]. In addition, arm swelling defined by an objective measure of interlimb volume or circumference differences) and lymphedema severity (defined by Common Toxicity Criteria) were less correlated with quality of life than arm symptoms (e.g., arm pain and swelling) [19]. Therefore, lymphatic pain and swelling are patient-centered health outcomes that are critical for monitoring the risk of and treating cancer-related lymphedema [20].

Obesity, defined as a BMI $\geq 30 \mathrm{~kg} / \mathrm{m}^{2}$, is a known risk factor for arm swelling or lymphedema defined as an increased limb size or girth comparing affected (or lymphedematous) and unaffected limbs [21,22]. However, it is unknown whether obesity is associated with patient-centered health outcomes of lymphatic pain, arm swelling, and truncal swelling. While some studies $[23,24]$ found that breast cancer patients with obesity were more likely to develop chronic bodily pain than those with a normal weight, others found no significant differences in the level of bodily pain between breast cancer patients with obesity and those with normal weight [21]. No studies have investigated the effects of obesity on patient-centered outcomes of lymphatic pain, arm swelling and truncal swelling in breast cancer patients. Therefore, the purpose of this study was to quantify the effects of obesity defined as a BMI $\geq 30 \mathrm{~kg} / \mathrm{m}^{2}$ on lymphatic pain, arm swelling, and truncal swelling in breast cancer patients. We hypothesized that breast cancer patients with a BMI $\geq 30 \mathrm{~kg} / \mathrm{m}^{2}$ would have higher odds of having lymphatic pain, arm swelling, and truncal swelling than those with a BMI $<30 \mathrm{~kg} / \mathrm{m}^{2}$ controlling for clinical and demographic characteristics.

\section{Materials and Methods}

\subsection{Study Design}

A cross-sectional and observational design was used.

\subsection{Ethical Considerations}

This study (IRB \# 16-01665) was approved by the Institutional Review Board of NYU Langone Health in New York City of the United States. All the participants signed the written informed consent.

\subsection{Setting}

The study was conducted in the breast cancer clinic of NYU Perlmutter Cancer Center, a National Cancer Institute designated cancer center in New York City of the United States. 


\subsection{Study Population}

The study population was women who were older than 21 years of age, had completed acute treatment (i.e., surgery, radiotherapy, chemotherapy) for breast cancer greater than three months before enrolling in the study, and had no signs of metastatic disease, recurrence, or other bulk diseases. We recruited 570 breast cancer survivors between December 2016 and March 2020. Patients were excluded from the study if data regarding BMI, body fat percentage, and body fat mass were incomplete. A final sample of 554 patients was included in this study. Figure 1 presents Patient Flowchart.

\section{Enrollment}

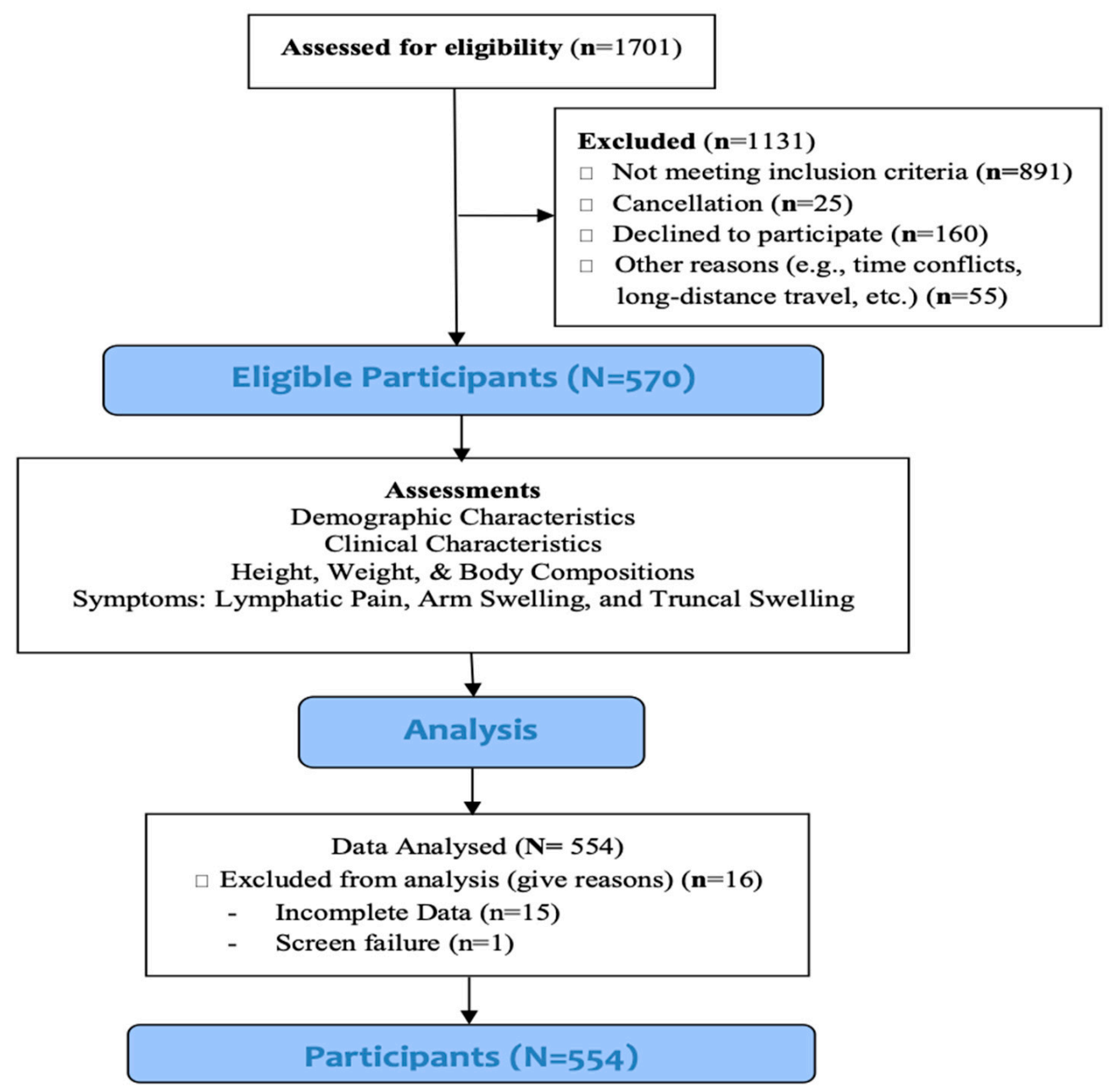

Figure 1. Patient flowchart.

\subsection{Variables and Measures}

\subsubsection{Obesity}

Anthropometric measurements were used to assess height, BMI, body fat percentage, and body fat mass. Height was measured without shoes to the nearest $0.1 \mathrm{~cm}$ using a digital stadiometer (Seca Corporation, Chino, CA, USA). A stand-on bioimpedance analysis (BIA) device (InBody 520, Biospace Co., Ltd., Cerritos, CA, USA) was used to measure weight without shoes to the nearest $0.05 \mathrm{~kg}$ and the device automatically calculated BMI (kilogram/meters squared, $\mathrm{kg} / \mathrm{m}^{2}$ ). Obesity was defined as a BMI $\geq 30 \mathrm{~kg} / \mathrm{m}^{2}$ [1]. The Inbody 520 multifrequency BIA device was also used to measure body fat percentage, and body fat mass using impedance at 5, 50, and $500 \mathrm{kHz}$. 


\subsubsection{Lymphatic Pain, Arm Swelling, and Truncal Swelling}

The Breast Cancer and Lymphedema Symptom Experience Index (BCLE-SEI) Part $\mathrm{I}$, a reliable and valid self-report instrument, was used to assess self-reported lymphatic pain, arm swelling, and truncal swelling $[25,26]$. Adequate internal consistency was demonstrated with a Cronbach's alpha of 0.92 for symptom occurrence. The discriminant validity of the instrument was supported by a significant difference in symptom occurrence $(\mathrm{z}=-6.938, p<0.001)$ between breast cancer patients with and without lymphedema. A response frame of the past three months was used to ensure that the symptoms were persistent and chronic. Each item was rated on a 5-point Likert scale (i.e., $0=$ no presence of a given symptom to $4=$ greatest severity of a given symptom). Arm swelling is defined and operationalized as the report of swelling in the affected ipsilateral upper limb. Lymphatic pain was defined as the co-occurrence of pain and swelling in the affected ipsilateral upper limb following breast cancer treatment. We operationalized lymphatic pain as the report of co-occurring pain sensations (i.e., pain, aching, or soreness) and arm swelling. Truncal swelling was defined as swelling in the upper body and operationalized as the report of co-occurring swelling in breast(s) and chest walls.

\subsubsection{Demographic and Clinical Data}

Demographic data were collected to include age, education, marital status, employment status, and ethnicity. Financial status was assessed by asking patients to respond to the questions regarding comfortable finance (i.e., having more than enough to make ends meet), adequate finance (i.e., having enough to make ends meet), and financial hardship (i.e., not having enough to make ends meet). Medical records were reviewed to obtain information on breast cancer diagnosis, stage of the disease, cancer location, types of surgery, lymph node procedures, types of adjuvant therapy (e.g., radiotherapy, chemotherapy, hormonal therapy), diabetes, and lymphedema diagnosis.

\subsection{Study Procedures and Data Collection}

All measures were completed during a single visit to NYU Perlmutter Cancer Center. To ensure accurate measures using the Inbody 520 multifrequency BIA device, patients were instructed to stay hydrated; not participate in vigorous weightlifting, aerobic exercise, or hot yoga; not use a sauna; and not consume alcohol during the $24 \mathrm{~h}$ prior to the scheduled research appointment. On the day of the visit, patients were instructed not to wear body oils, lotions, or jewelry. Patients were also instructed to limit exercise to leisure walking, consume no caffeine or food (water was encouraged), and to remove any compression garments two hours prior to the appointment. All the self-reported questionnaires were administered to the patients during the visit via a study iPad connected to the study-specific electronic database capture system.

\subsection{Data Analysis}

Data were analyzed using Stata 16 SE. Descriptive statistics were calculated for demographic and clinical characteristics. Continuous variables were summarized in terms of means, standard deviations (SD), and ranges. Categorical variables were summarized using frequencies and proportions. Confidence intervals (CI) were reported to quantify the precision of estimates.

Patients were classified into obesity (BMI $\geq 30 \mathrm{~kg} / \mathrm{m}^{2}$ ) and non-obesity (BMI $<30 \mathrm{~kg} / \mathrm{m}^{2}$ ) groups. Group differences in demographic and clinical characteristics were estimated using $t$-test for continuous and Pearson $\chi^{2}$ test for categorical data. Effect sizes were calculated for differences between groups (i.e., Cohen's $d$ for continuous variables and Cramer's $V$ for categorical variables).

Multivariable logistic regression models were used to estimate the odds ratio (OR) with $95 \%$ confidence interval (CI) to quantify the effects of obesity on lymphatic pain, arm swelling, and truncal swelling controlling for clinical and demographic characteristics as well as body fat percentage. Clinical characteristics selected in the regression analyses 
were based on known risk factors for lymphedema and chronic cancer pain [17-25]. These variables included types of cancer surgery (mastectomy versus lumpectomy), types of lymph node procedures (sentinel lymph node biopsy versus axillary lymph node dissection), number of lymph nodes removed, receipt of radiotherapy, receipt of chemotherapy, receipt of hormonal therapy, years elapsed since breast cancer treatment, history of diabetes, and lymphedema diagnosis. Demographic characteristics were also included in the regression analysis. Body fat percentage was included in the regression models since body fat percentage and body fat mass are considered possible indicators for obesity [3]. In addition, we were interested in the effects of obesity (BMI $\geq 30 \mathrm{~kg} / \mathrm{m}^{2}$ ) after controlling for body fat in relation to muscle mass (i.e., body fat percentage). Collinearity between obesity (BMI $\geq 30 \mathrm{~kg} / \mathrm{m}^{2}$ ) and body fat percentage were evaluated and had acceptable low levels of variance inflation $\left(V I F_{\text {Obesity }}=1.91 ; V F_{\text {Body fat percentage }}=1.92\right)$. Body fat mass was not included in the regression models due to strong collinearity between body fat percentage and body fat mass as evidenced by the variance inflation $\left(V I F_{\text {Body fat percentage }}=9.87\right.$; $V I F_{\text {Body fat mass }}=7.16$ ). All the tests were conducted at 0.05 alpha level and $95 \%$ confidence interval (CI). In addition to reporting the significance of parameters, model fitness was evaluated by McFadden's pseudo $\mathrm{R}^{2}$ and a link-test for the logistic regression models. We present the regression parameters in exponentiated form, which are interpreted as odds ratios. Odds ratios are a measure of effect size, with an odds ratio equal to exactly 1 indicating no effect [27]. Subsequent regression models were used to examine the specific associations between BMI and the three outcomes among patients who were obese (BMI $\geq 30 \mathrm{~kg} / \mathrm{m}^{2}$ ), using scatterplots to inform the possible linear or polynomial trends to be tested.

\section{Results}

\subsection{Demographic and Clinical Characteristics of the Participants}

As shown in Table 1, the sample of 554 patients in the study had a mean age of 57.96 years $(\mathrm{SD}=11.31$; range $=26-85)$. Among these patients, $73.65 \%$ had a bachelor's or graduate degree, $59.21 \%$ were married or partnered, and $64.8 \%$ were employed. In terms of financial status, $61.91 \%$ had comfortable finance of having more than enough to make ends meet and $31.77 \%$ had adequate finance of having enough to make ends meet, but $6.32 \%$ had financial hardship of not having enough to make ends meet. While $70.76 \%$ of patients were White, 29.24\% were non-white: $8.84 \%(n=49)$ being Asian, 5.23\% $(n=29)$ black or African American, and 15.2\% $(n=84)$ Hispanic.

Table 1. Demographic characteristics of participants $(N=554)$.

\begin{tabular}{|c|c|c|c|c|c|c|c|}
\hline \multirow[b]{2}{*}{$\begin{array}{l}\text { Continuous } \\
\text { Variables }\end{array}$} & \multirow{2}{*}{$\begin{array}{l}\text { All Sample } \\
\mathrm{N}=554 \\
\begin{array}{c}\text { Mean } \pm \mathrm{SD}^{1} \\
\text { (Range) }\end{array}\end{array}$} & \multirow{2}{*}{$\begin{array}{c}\text { Obesity } \\
\text { BMI } \geq 30 \mathrm{~kg} / \mathrm{m}^{2} \\
\mathrm{n}=135\end{array}$} & \multirow{2}{*}{$\begin{array}{c}\text { Non-Obesity } \\
\text { BMI }<30 \mathrm{~kg} / \mathrm{m}^{2} \\
\mathrm{n}=419\end{array}$} & \multicolumn{4}{|c|}{ Test Statistics } \\
\hline & & & & $\mathbf{t}$ & $\mathrm{df}^{1}$ & $p$ (t-Test) & Effect Size $^{2}$ \\
\hline Age (in years) & $\begin{array}{c}57.96 \pm 11.31 \\
(26-85)\end{array}$ & $\begin{array}{l}57.50 \pm 11.31 \\
\quad(32-82)\end{array}$ & $\begin{array}{l}58.11 \pm 11.32 \\
\quad(26-85)\end{array}$ & 0.54 & 552 & 0.5916 & 0.05 \\
\hline Body fat percentage & $\begin{array}{c}34.71 \pm 8.96 \\
(7.8-55.2)\end{array}$ & $\begin{array}{c}45.17 \pm 4.90 \\
(29.2-55.2)\end{array}$ & $\begin{array}{c}31.34 \pm 7.20 \\
(7.8-51.1)\end{array}$ & -20.82 & 552 & $<0.001$ & -2.06 \\
\hline Body fat mass & $\begin{array}{c}56.52 \pm 25.93 \\
(7.5-182.3)\end{array}$ & $\begin{array}{c}92.07 \pm 20.44 \\
(54.4-182.3)\end{array}$ & $\begin{array}{c}45.07 \pm 14.69 \\
(7.5-83.7)\end{array}$ & -29.18 & 552 & $<0.001$ & -2.89 \\
\hline Ethnicity & & & & 12.92 & 1 & $<0.001$ & -0.15 \\
\hline Non-white & $162(29.24)$ & $56(41.48)$ & $106(25.30)$ & & & & \\
\hline White & $392(70.76)$ & $79(58.52)$ & $313(74.70)$ & & & & \\
\hline Marital status & & & & 0.03 & 1 & 0.852 & -0.01 \\
\hline Married/partnered & $328(59.21)$ & 79 (58.52) & $249(59.43)$ & & & & \\
\hline Not married & $226(40.79)$ & $56(41.48)$ & $170(40.57)$ & & & & \\
\hline
\end{tabular}


Table 1. Cont.

\begin{tabular}{|c|c|c|c|c|c|c|c|}
\hline $\begin{array}{l}\text { Categorical } \\
\text { Variables }\end{array}$ & n (\%) & n (\%) & n $(\%)$ & Pearson $\chi^{2}$ & df & $p\left(\chi^{2}\right)$ & Effect Size \\
\hline Education & & & & 2.73 & 2 & 0.255 & 0.07 \\
\hline $\begin{array}{l}\text { Associate Degree } \\
\text { or less }\end{array}$ & $146(26.35)$ & $41(30.37)$ & $105(25.06)$ & & & & \\
\hline Bachelor's Degree & 209 (37.73) & $53(39.26)$ & $156(37.23)$ & & & & \\
\hline Graduate Degree & 199 (35.92) & $41(30.37)$ & $158(37.71)$ & & & & \\
\hline $\begin{array}{l}\text { Financial status } \\
\text { Comfortable }\end{array}$ & & & & 16.52 & 2 & $<0.001$ & 0.17 \\
\hline $\begin{array}{l}\text { Finance: Have more } \\
\text { than enough to make } \\
\text { ends meet } \\
\text { Adequate Finance: }\end{array}$ & $343(61.91)$ & $68(50.37)$ & $275(65.63)$ & & & & \\
\hline $\begin{array}{l}\text { Have enough to } \\
\text { make ends meet } \\
\text { Financial }\end{array}$ & $176(31.77)$ & $50(37.04)$ & $126(30.07)$ & & & & \\
\hline $\begin{array}{l}\text { Hardship: Do not } \\
\text { have enough to make } \\
\text { ends meet }\end{array}$ & $35(6.32)$ & 17 (12.59) & $18(4.30)$ & & & & \\
\hline Employment status & & & & 0.01 & 1 & 0.920 & -0.004 \\
\hline Employed & $359(64.80)$ & $87(64.44)$ & $272(64.92)$ & & & & \\
\hline Unemployed & $195(35.20)$ & $48(35.56)$ & $147(35.08)$ & & & & \\
\hline
\end{tabular}

${ }^{1}$ SD: Standard Deviation; df: degree of freedom; ${ }^{2}$ Effect size: Cohen's $d$ for continuous variables and Cramer's $V$ for categorical variables.

In terms of clinical characteristics (Table 2), 46.93\% of the patients had a lumpectomy, $53.07 \%$ a mastectomy, $64.08 \%$ chemotherapy, $72.2 \%$ radiotherapy, and $83.57 \%$ hormonal therapy. While $59.21 \%$ of the patients underwent axillary lymph node dissection, $40.79 \%$ had only sentinel lymph node biopsy. The mean lymph nodes removed was $8.64(\mathrm{SD}=8.41$; range $=1-35)$. The mean years elapsed since the breast cancer treatment was $4.75(\mathrm{SD}=5.59$; range $=0-43$ years). Among the 554 patients, only $3.97 \%$ had a history of diabetes, while $22.02 \%$ patients had a diagnosis of lymphedema. Between-groups effects sizes for demographic and clinical characteristics are presented in Tables 1 and 2.

Table 2. Clinical characteristics of participants $(N=554)$.

\begin{tabular}{|c|c|c|c|c|c|c|c|}
\hline & $\begin{array}{l}\text { All Sample } \\
N=554\end{array}$ & $\begin{array}{c}\text { Obesity } \\
\text { BMI } \geq 30 \mathrm{~kg} / \mathrm{m}^{2}\end{array}$ & $\begin{array}{l}\text { Non-Obesity } \\
\text { BMI }<30 \mathrm{~kg} / \mathrm{m}^{2}\end{array}$ & \multicolumn{4}{|c|}{ Test Statistics } \\
\hline Continuous Variables & $\begin{array}{l}\text { Mean } \pm \text { SD }^{1} \\
\quad \text { (Range) }\end{array}$ & $\begin{array}{l}\text { Mean } \pm \text { SD } \\
\text { (Range) }\end{array}$ & $\begin{array}{l}\text { Mean } \pm \text { SD } \\
\text { (Range) }\end{array}$ & $\mathbf{t}$ & $\mathrm{df}^{1}$ & $p(t$-Test $)$ & Effect Size $^{2}$ \\
\hline $\begin{array}{l}\text { Years elapsed since the } \\
\text { breast cancer treatment }\end{array}$ & $\begin{array}{l}4.75 \pm 5.59 \\
\quad(0-43)\end{array}$ & $\begin{array}{l}4.37 \pm 5.10 \\
\quad(0-27)\end{array}$ & $\begin{array}{l}4.87 \pm 5.72 \\
\quad(0-43)\end{array}$ & 0.89 & 552 & 0.3743 & 0.09 \\
\hline $\begin{array}{l}\text { Number of lymph nodes } \\
\text { removed }\end{array}$ & $\begin{array}{l}8.64 \pm 8.41 \\
\quad(1-35)\end{array}$ & $\begin{array}{c}8.44 \pm 7.44 \\
(1-35)\end{array}$ & $\begin{array}{c}8.70 \pm 8.71 \\
(1-35)\end{array}$ & 0.32 & 552 & 0.7508 & 0.03 \\
\hline Categorical Variables & n (\%) & n (\%) & n (\%) & $\begin{array}{c}\text { Pearson } \\
\chi^{2}\end{array}$ & df & $p\left(\chi^{2}\right)$ & Effect Size \\
\hline Lymphedema diagnosis & & & & 7.25 & 1 & 0.007 & 0.11 \\
\hline Yes & $122(22.02)$ & $41(30.37)$ & 81 (19.33) & & & & \\
\hline No & $432(77.98)$ & $94(69.63)$ & $338(80.67)$ & & & & \\
\hline Diabetes & & & & 11.32 & 1 & 0.001 & 0.14 \\
\hline Yes & $22(3.97)$ & $12(8.89)$ & $10(2.39)$ & & & & \\
\hline No & $532(96.03)$ & $123(91.11)$ & $409(97.61)$ & & & & \\
\hline Lymph node procedures & & & & 1.50 & 1 & 0.221 & 0.05 \\
\hline $\begin{array}{l}\text { Axillary lymph node } \\
\text { dissection }\end{array}$ & $328(59.21)$ & $86(63.70)$ & $242(57.76)$ & & & & \\
\hline $\begin{array}{l}\text { Sentinel lymph node } \\
\text { biopsy }\end{array}$ & $226(40.79)$ & $49(36.30)$ & $177(42.24)$ & & & & \\
\hline
\end{tabular}


Table 2. Cont.

\begin{tabular}{cccccc}
\hline $\begin{array}{c}\text { Radiotherapy } \\
\text { Yes }\end{array}$ & $400(72.20)$ & $103(76.30)$ & $297(70.88)$ & 1.49 & 0.05 \\
No & $154(27.80)$ & $32(23.70)$ & $122(29.12)$ & $\mathbf{0 . 5 2}$ \\
Yes & $355(64.08)$ & $90(66.67)$ & $265(63.25)$ & $\mathbf{0 . 4 7 1}$ \\
No & $199(35.92)$ & $45(33.33)$ & $154(36.75)$ & $\mathbf{0 . 1 0}$ & $\mathbf{1}$ \\
Yes & $463(83.57)$ & $114(84.44)$ & $349(83.29)$ & $\mathbf{0 . 7 5 4}$ \\
No & $91(16.43)$ & $21(15.56)$ & $70(16.71)$ & \\
\hline
\end{tabular}

${ }^{1}$ SD: Standard Deviation; df: degree of freedom; ${ }^{2}$ Effect size: Cohen's $d$ for continuous variables and Cramer's $V$ for categorical variables.

\subsection{Obesity, Body Fat Percentage and Body Fat Mass}

The average BMI of the total patient sample $(n=554)$ was $26.69(\mathrm{SD}=5.80$; range $=16-58.6)$. Among the 554 patients, $24.36 \%(n=135)$ were obese (BMI $\left.\geq 30 \mathrm{~kg} / \mathrm{m}^{2}\right)$ and $75.63 \%(n=419)$ non-obese $\left(\mathrm{BMI}<30 \mathrm{~kg} / \mathrm{m}^{2}\right)$. The average BMI for patients with obesity was $34.97(\mathrm{SD}=4.40$; range $=30-58.6)$ while the average BMI for patients with non-obesity was 24.02 ( $S D=3.01$; range $=16-29.9)$. Patients with obesity were more likely to be non-white $\left(\chi^{2}(1)=12.92\right.$, $p<0.001 ; V=-0.15)$, have diabetes $\left(\chi^{2}(1)=11.32, p<0.001 ; V=0.14\right)$, have a diagnosis of lymphedema $\left(\chi^{2}(1)=7.25, p<0.007 ; V=0.11\right)$, and have financial hardship $\left(\chi^{2}(1)=16.52\right.$, $p<0.001 ; V=0.17)$.

As shown in Table 1, the average body fat percentage for the total patient sample $(N=554)$ was $34.71(S D=8.96$; range $=7.8-55.2)$. Significantly higher body fat percentage $(t(552)=-20.82 ; p<0.001 ; d=-2.06)$ was found in patients with obesity $(M=45.17$; $\mathrm{SD}=4.90 ;$ range $=29.2-55.2)$ compared to patients with non-obesity $(M=31.34 ; \mathrm{SD}=7.20$; range $=7.8-51.1)$. The average body fat mass for the total patient sample $(N=554)$ was 56.52 $(S D=25.93$; range $=7.5-182.3)$. Significantly higher body fat mass $(t(552)=-29.18, p<0.001$; $d=-2.89)$ was found in patients with obesity $(M=92.07 ; S D=20.44 ;$ range $=54.4-182.3)$ compared to patients with non-obesity $(M=45.07 ; \mathrm{SD}=14.69$; range $=7.5-83.7)$.

\subsection{The Effect of Obesity on Lymphatic Pain}

As shown in Table 3, among the 554 patients, 32.85\% experienced lymphatic pain. Significantly more patients with obesity reported lymphatic pain compared to those with non-obesity $\left(51.85 \%\right.$ vs. $\left.26.73 ; \chi^{2}(1)=29.21, p<0.001\right)$.

As shown in Table 4, to evaluate the effect of obesity on the presence of lymphatic pain given clinical and demographic characteristics as well as body fat percentage, a multivariable logistic regression model fits the data well (McFadden's Pseudo $\mathrm{R}^{2}=0.137$; LR $\left.\chi^{2}(18)=95.77 ; p<0.001\right)$. Controlling for clinical and demographic characteristics as well as body fat percentage, obesity $(\mathrm{B}=1.25 ; z=3.94 ; \mathrm{OR}=3.49 ; 95 \% \mathrm{CI} 1.87-6.50 ; p<0.001)$ had the greatest effect on lymphatic pain. Other covariates predicted lymphatic pain, including having financial hardship $(\mathrm{B}=1.16 ; z=2.74 ; \mathrm{OR}=3.19 ; 95 \% \mathrm{CI} 1.39-7.31 ; p=0.006)$, having radiotherapy $(\mathrm{B}=0.58 ; z=2.13 ; \mathrm{OR}=1.78 ; 95 \% \mathrm{CI} 1.05-3.03 ; p=0.033)$, and having more lymph nodes removed $(B=0.03 ; z=2.05 ; O R=1.03 ; 95 \% C I 1.00-1.06 ; p=0.041)$. Subsequent analysis within the obesity group suggests that there is a linear relationship between BMI and lymphatic pain scores amongst patients with a $\mathrm{BMI} \geq 30 \mathrm{~kg} / \mathrm{m}^{2}(\mathrm{~B}=0.28 ; 95 \% \mathrm{CI}$ $0.05-0.41 ; t=2.50 ; p=0.014$ ). For obese patients, as BMI increases, levels of lymphatic pain are expected to increase linearly.

\subsection{The Effect of Obesity on Arm Swelling}

As shown in Table 3, among the 554 patients, 39.17\% experienced arm swelling. Significantly more patients with obesity reported arm swelling compared to those with non-obesity $\left(57.04 \%\right.$ vs. $\left.33.41 ; \chi^{2}(1)=23.92 ; p<0.001\right)$. As shown in Table 5 , to evaluate the effects of obesity on the presence of arm swelling given clinical and demographic characteristics as well as body fat percentage, a multivariable logistic regression model fits the data well (McFadden's Pseudo $\mathrm{R}^{2}=0.142 ; \mathrm{LR}^{2}(18)=105.47 ; p<0.001$ ). Controlling for clinical and demographic characteristics as well as body fat percentage, obesity $(\mathrm{B}=1.38$; 
$z=4.40 ;$ OR $=3.98 ; 95 \%$ CI 1.82-4.43; $p<0.001)$ had the greatest effect on arm swelling. Other significant predictors included having financial hardship $(\mathrm{B}=0.89 ; z=2.03 ; \mathrm{OR}=2.43$; $95 \%$ CI 1.03-5.74; $p=0.043)$, having mastectomy $(\mathrm{B}=0.78 ; z=3.13 ; \mathrm{OR}=2.17 ; 95 \% \mathrm{CI}$ 1.40-3.53; $p=0.002)$, having axillary lymph node dissection $(\mathrm{B}=0.53 ; z=2.06 ; \mathrm{OR}=1.69$; $95 \%$ CI 1.03-2.80; $p=0.040)$, having radiotherapy $(\mathrm{B}=0.80 ; z=3.03 ; \mathrm{OR}=2.23 ; 95 \% \mathrm{CI}$ $1.33-3.75] ; p=0.002)$, and having more lymph nodes removed $(\mathrm{B}=0.03 ; z=2.05 ; \mathrm{OR}=1.03$; $95 \%$ CI 1.00-1.06; $p=0.040$ ). Subsequent regression analysis did not reveal any additional effects of BMI on degree of arm swelling among patients with a BMI $\geq 30 \mathrm{~kg} / \mathrm{m}^{2}$. In other words, patients with a BMI $\geq 30 \mathrm{~kg} / \mathrm{m}^{2}$ exhibited similar levels of arm swelling, regardless of specific BMI values.

Table 3. Differences in lymphatic pain, arm swelling and truncal swelling between women with obesity and non-obesity $(N=554)$.

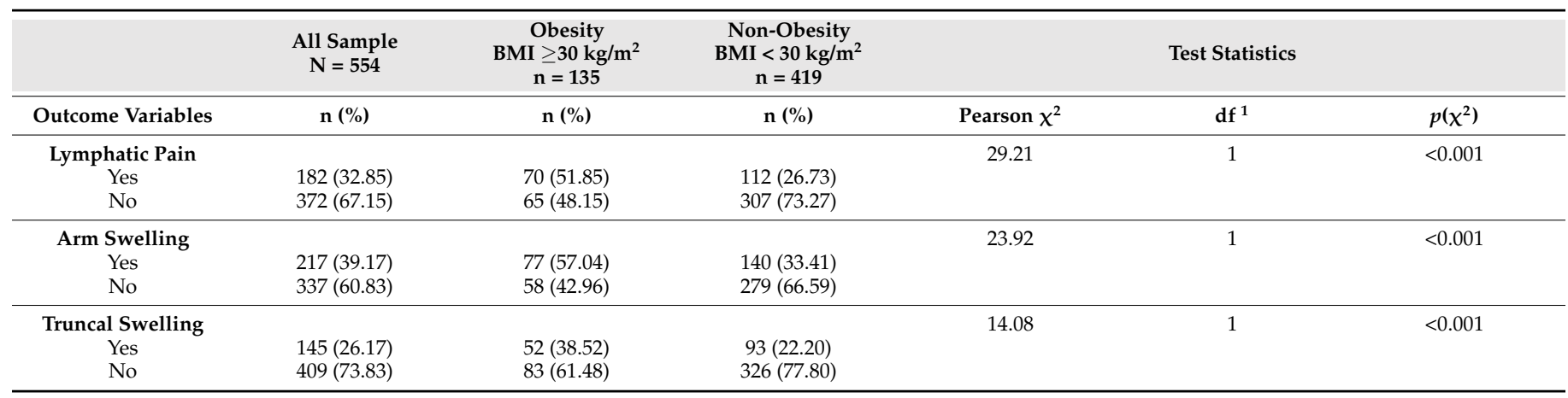

${ }^{1} \mathrm{df}$ : degree of freedom.

Table 4. Multivariable logistic regression for lymphatic pain $(N=554)$.

\begin{tabular}{|c|c|c|c|c|c|c|}
\hline & \multicolumn{6}{|c|}{ Multivariable Logistic Regression } \\
\hline & $\mathbf{B}^{1}$ & SE & z-Value & Odds Ratio & OR $95 \%$ CI & $p$ Value \\
\hline \multicolumn{7}{|l|}{ Obesity } \\
\hline Yes & 1.25 & 0.32 & 3.94 & 3.49 & $(1.87,6.50)$ & $<0.001$ \\
\hline No & - & - & - & - & - & \\
\hline \multicolumn{7}{|l|}{ Diabetes } \\
\hline Yes & 0.27 & 0.49 & 0.55 & 1.31 & $(0.50,3.43)$ & 0.583 \\
\hline No & - & - & - & - & - & \\
\hline \multicolumn{7}{|l|}{ Ethnicity } \\
\hline Non-white & -0.20 & 0.23 & -0.89 & 0.82 & $(0.53,1.27)$ & 0.374 \\
\hline White & - & - & - & - & - & \\
\hline \multicolumn{7}{|l|}{ Marital status } \\
\hline Married/partnered & -0.15 & 0.21 & -0.70 & 0.86 & $(0.57,1.31)$ & 0.483 \\
\hline Not married & - & - & - & - & - & \\
\hline \multicolumn{7}{|l|}{ Education } \\
\hline Associate Degree or less & 0.42 & 0.26 & 1.60 & 1.52 & $(0.91,2.54)$ & 0.110 \\
\hline Bachelor's degree & - & - & - & - & - & \\
\hline$\geq$ Master's Degree & -0.13 & 0.24 & -0.54 & 0.88 & $(0.55,1.41)$ & 0.592 \\
\hline \multicolumn{7}{|l|}{ Financial status } \\
\hline Comfortable finance & 0.16 & 0.22 & 0.71 & 1.17 & $(0.76,1.81)$ & 0.477 \\
\hline Financial hardship & 1.16 & 0.42 & 2.74 & 3.19 & $(1.39,7.31)$ & 0.006 \\
\hline Adequate finance & - & - & - & - & - & \\
\hline \multicolumn{7}{|l|}{ Employment status } \\
\hline Employed & 0.12 & 0.23 & 0.52 & 1.13 & $(0.72,1.79)$ & 0.600 \\
\hline Unemployed & - & - & - & - & - & \\
\hline \multicolumn{7}{|l|}{ Types of surgery } \\
\hline Mastectomy & 0.40 & 0.25 & 1.60 & 1.50 & $(0.91,2.45)$ & 0.109 \\
\hline Lumpectomy & - & - & - & - & - & \\
\hline Lymph node procedures & & & & & & \\
\hline Axillary lymph node dissection & 0.42 & 0.27 & 1.57 & 1.52 & $(0.90,2.56)$ & 0.117 \\
\hline Sentinel lymph node biopsy & - & - & - & - & - & \\
\hline
\end{tabular}


Table 4. Cont.

\begin{tabular}{|c|c|c|c|c|c|c|}
\hline & \multicolumn{6}{|c|}{ Multivariable Logistic Regression } \\
\hline & $\mathrm{B}^{1}$ & SE & z-Value & Odds Ratio & OR $95 \%$ CI & $p$ Value \\
\hline \multicolumn{7}{|l|}{ Radiotherapy } \\
\hline Yes & 0.58 & 0.27 & 2.13 & 1.78 & $(1.05,3.03)$ & 0.033 \\
\hline No & - & - & - & - & - & \\
\hline \multicolumn{7}{|l|}{ Chemotherapy } \\
\hline Yes & 0.33 & 0.24 & 1.36 & 1.39 & $(0.87,2.24)$ & 0.173 \\
\hline No & - & - & - & - & - & \\
\hline \multicolumn{7}{|l|}{ Hormonal therapy } \\
\hline Yes & 0.52 & 0.30 & 1.74 & 1.69 & $(0.94,3.04)$ & 0.082 \\
\hline No & - & - & - & - & - & \\
\hline Age (in years) & -0.02 & 0.01 & -1.47 & 0.98 & $(0.96,1.01)$ & 0.142 \\
\hline $\begin{array}{l}\text { Years elapsed since the breast } \\
\text { cancer treatment }\end{array}$ & -0.02 & 0.02 & -0.99 & 0.98 & $(0.94,1.02)$ & 0.323 \\
\hline $\begin{array}{l}\text { Numbers of lymph nodes } \\
\text { removed }\end{array}$ & 0.03 & 0.02 & 2.05 & 1.03 & $(1.00,1.06)$ & 0.041 \\
\hline Body fat percentage & -0.01 & 0.02 & -0.94 & 0.99 & $(0.96,1.02)$ & 0.348 \\
\hline Intercept & -2.29 & 0.67 & -3.40 & 0.10 & $(0.03,0.38)$ & 0.001 \\
\hline McFadden's Pseudo $\mathrm{R}^{2}$ & \multicolumn{6}{|c|}{0.137} \\
\hline $\operatorname{LR} \chi^{2}(18)$ & \multicolumn{6}{|c|}{95.77} \\
\hline Prob $>\chi^{2}$ & \multicolumn{6}{|c|}{$<0.001$} \\
\hline
\end{tabular}

Table 5. Multivariable logistic regression for arm swelling $(N=554)$.

\begin{tabular}{|c|c|c|c|c|c|c|}
\hline & \multicolumn{6}{|c|}{ Multivariable Logistic Regression } \\
\hline & $\mathbf{B}^{1}$ & SE & z-Value & Odds Ratio & OR $95 \%$ CI & $p$ Value \\
\hline \multicolumn{7}{|l|}{ Obesity } \\
\hline Yes & 1.38 & 0.31 & 4.40 & 3.98 & $(1.82,4.43)$ & $<0.001$ \\
\hline No & - & - & - & - & - & \\
\hline \multicolumn{7}{|l|}{ Diabetes } \\
\hline Yes & 0.20 & 0.50 & 0.39 & 1.22 & $(0.46,3.22)$ & 0.695 \\
\hline No & - & - & - & - & - & \\
\hline \multicolumn{7}{|l|}{ Ethnicity } \\
\hline Non-white & -0.09 & 0.22 & -0.39 & 0.92 & $(0.60,1.41)$ & 0.696 \\
\hline White & - & - & - & - & - & \\
\hline \multicolumn{7}{|l|}{ Marital status } \\
\hline Married/partnered & -0.20 & 0.20 & -0.97 & 0.82 & $(0.55,1.22)$ & 0.330 \\
\hline Not married & - & - & - & - & - & \\
\hline \multicolumn{7}{|l|}{ Education } \\
\hline Associate Degree or less & 0.35 & 0.26 & 1.34 & 1.41 & $(0.85,2.34)$ & 0.179 \\
\hline Bachelor's degree & - & - & - & - & - & \\
\hline$\geq$ Master's Degree & -0.12 & 0.23 & -0.51 & 0.89 & $(0.56,1.40)$ & 0.612 \\
\hline \multicolumn{7}{|l|}{ Financial Status } \\
\hline Comfortable finance & -0.04 & 0.22 & -0.19 & 0.96 & $(0.63,1.47)$ & 0.848 \\
\hline Financial hardship & 0.89 & 0.44 & 2.03 & 2.43 & $(1.03,5.74)$ & 0.043 \\
\hline Adequate finance & - & - & - & - & - & \\
\hline \multicolumn{7}{|l|}{ Employment status } \\
\hline Employed & 0.002 & 0.22 & 0.01 & 1.00 & $(0.65,1.56)$ & 0.992 \\
\hline Unemployed & - & - & - & - & - & \\
\hline \multicolumn{7}{|l|}{ Types of surgery } \\
\hline Mastectomy & 0.78 & 0.25 & 3.13 & 2.17 & $(1.4,3.53)$ & 0.002 \\
\hline Lumpectomy & - & - & - & - & - & \\
\hline
\end{tabular}


Table 5. Cont.

\begin{tabular}{|c|c|c|c|c|c|c|}
\hline & \multicolumn{6}{|c|}{ Multivariable Logistic Regression } \\
\hline & $\mathbf{B}^{1}$ & SE & z-Value & Odds Ratio & OR 95\% CI & $p$ Value \\
\hline \multicolumn{7}{|l|}{ Lymph node procedures } \\
\hline $\begin{array}{l}\text { Axillary lymph node } \\
\text { dissection }\end{array}$ & 0.53 & 0.26 & 2.06 & 1.69 & $(1.03,2.80)$ & 0.040 \\
\hline Sentinel lymph node biopsy & - & - & - & - & - & \\
\hline \multicolumn{7}{|l|}{ Radiotherapy } \\
\hline Yes & 0.80 & 0.26 & 3.03 & 2.23 & $(1.33,3.75)$ & 0.002 \\
\hline No & - & - & - & - & - & \\
\hline \multicolumn{7}{|l|}{ Chemotherapy } \\
\hline Yes & 0.17 & 0.23 & 0.76 & 1.19 & $(0.76,1.87)$ & 0.448 \\
\hline No & - & - & - & - & - & \\
\hline \multicolumn{7}{|l|}{ Hormonal therapy } \\
\hline Yes & -0.02 & 0.27 & -0.06 & 0.98 & $(0.58,1.67)$ & 0.948 \\
\hline No & - & - & - & - & - & \\
\hline Age (in years) & -0.01 & 0.01 & -0.50 & 0.99 & $(0.97,1.02)$ & 0.620 \\
\hline $\begin{array}{l}\text { Years elapsed since the } \\
\text { breast cancer treatment }\end{array}$ & -0.003 & 0.02 & -0.19 & 1.00 & $(0.96,1.03)$ & 0.852 \\
\hline $\begin{array}{l}\text { Number of lymph nodes } \\
\text { removed }\end{array}$ & 0.03 & 0.02 & 2.05 & 1.03 & $(1.00,1.06)$ & 0.040 \\
\hline Body fat percentage & -0.03 & 0.02 & -1.93 & 0.97 & $(0.94,1.00)$ & 0.053 \\
\hline Intercept & -1.20 & 0.62 & -1.94 & 0.30 & $(0.09,1.01)$ & 0.052 \\
\hline McFadden's Pseudo $\mathrm{R}^{2}$ & \multicolumn{6}{|c|}{0.142} \\
\hline $\operatorname{LR} \chi^{2}(18)$ & \multicolumn{6}{|c|}{105.47} \\
\hline Prob $>\chi^{2}$ & \multicolumn{6}{|c|}{$<0.001$} \\
\hline
\end{tabular}

${ }^{1}$ B: Regression Coefficient; SE: Standard Error; CI: Confidence Interval; OR: Odds Ratio; -: Reference Group.

\subsection{The Effect of Obesity on Truncal Swelling}

As shown in Table 3, among the 554 patients, 26.17\% experienced truncal swelling. Significantly more patients with obesity reported truncal swelling compared to those with non-obesity $\left(38.52 \%\right.$ vs. $\left.22.2 ; \chi^{2}(1)=14.08, p<0.001\right)$. As shown in Table 6 , to evaluate the effects of obesity on the presence of truncal swelling given clinical and demographic characteristics as well as body fat percentage, a multivariable logistic regression model fits the data well (McFadden's Pseudo $\mathrm{R}^{2}=0.060 ; \mathrm{LR}^{2}(18)=38.35 ; p=0.004$ ). Controlling for clinical and demographic characteristics as well as body fat percentage, obesity ( $\mathrm{B}=0.61$; $z=1.95 ; \mathrm{OR}=1.85 ; 95 \% \mathrm{CI} 1.00-3.42 ; p=0.051$ ) had no significant effect on truncal swelling. Significant predictors included having had a mastectomy $(\mathrm{B}=-0.64 ; z=-2.45 ; \mathrm{OR}=0.53$; $95 \%$ CI $0.32-0.88 ; p=0.014)$ and younger age $(B=-0.02 ; z=-2.10 ; \mathrm{OR}=0.98 ; 95 \% \mathrm{CI}$ $0.96-1.00 ; p=0.036$ ). In subsequent regression analysis, no additional effects of BMI on truncal swelling were observed among patients with a BMI $\geq 30 \mathrm{~kg} / \mathrm{m}^{2}$. The results suggest that patients with a BMI $\geq 30 \mathrm{~kg} / \mathrm{m}^{2}$ are expected to have similar levels of truncal swelling, regardless of specific BMI values.

Table 6. Multivariable logistic regression for truncal swelling $(N=554)$.

\begin{tabular}{|c|c|c|c|c|c|c|}
\hline & \multicolumn{6}{|c|}{ Multivariable Logistic Regression } \\
\hline & B $^{1}$ & SE & z-Value & Odds Ratio & OR 95\% CI & $p$ Value \\
\hline \multicolumn{7}{|c|}{ Obesity } \\
\hline Yes & 0.61 & 0.31 & 1.95 & 1.85 & $(1.00,3.42)$ & 0.051 \\
\hline No & - & - & - & - & - & \\
\hline \multicolumn{7}{|c|}{ Diabetes } \\
\hline Yes & 0.001 & 0.51 & 0.00 & 1.00 & $(0.37,2.70)$ & 0.997 \\
\hline No & - & - & - & - & - & \\
\hline
\end{tabular}


Table 6. Cont.

\begin{tabular}{|c|c|c|c|c|c|c|}
\hline & \multicolumn{6}{|c|}{ Multivariable Logistic Regression } \\
\hline & $\mathbf{B}^{1}$ & SE & z-Value & Odds Ratio & OR 95\% CI & $p$ Value \\
\hline \multicolumn{7}{|l|}{ Ethnicity } \\
\hline Non-white & -0.06 & 0.23 & -0.25 & 0.94 & $(0.60,1.48)$ & 0.805 \\
\hline White & - & - & - & - & - & \\
\hline \multicolumn{7}{|l|}{ Marital status } \\
\hline Married/partnered & -0.14 & 0.21 & -0.65 & 0.87 & $(0.57,1.32)$ & 0.514 \\
\hline Not married & - & - & - & - & - & \\
\hline \multicolumn{7}{|l|}{ Education } \\
\hline Associate Degree or less & 0.38 & 0.27 & 1.43 & 1.47 & $(0.87,2.48)$ & 0.152 \\
\hline Bachelor's degree & - & - & - & - & - & \\
\hline$\geq$ Master's Degree & 0.26 & 0.24 & 1.07 & 1.30 & $(0.80,2.09)$ & 0.286 \\
\hline \multicolumn{7}{|l|}{ Financial status } \\
\hline Comfortable finance & 0.30 & 0.22 & 1.35 & 1.35 & $(0.87,2.09)$ & 0.176 \\
\hline Financial hardship & 0.22 & 0.42 & 0.52 & 1.24 & $(0.55,2.81)$ & 0.602 \\
\hline Adequate finance & - & - & - & - & - & - \\
\hline \multicolumn{7}{|l|}{ Employment status } \\
\hline Employed & -0.04 & 0.23 & -0.16 & 0.96 & $(0.61,1.52)$ & 0.869 \\
\hline Unemployed & - & - & - & - & - & \\
\hline \multicolumn{7}{|l|}{ Types of surgery } \\
\hline Mastectomy & -0.64 & 0.26 & -2.45 & 0.53 & $(0.32,0.88)$ & 0.014 \\
\hline Lumpectomy & - & - & - & - & - & \\
\hline \multicolumn{7}{|l|}{ Lymph node procedures } \\
\hline Axillary lymph node dissection & 0.25 & 0.26 & 0.96 & 1.29 & $(0.77,2.17)$ & 0.337 \\
\hline Sentinel lymph node biopsy & - & - & - & - & - & \\
\hline \multicolumn{7}{|l|}{ Radiotherapy } \\
\hline Yes & 0.10 & 0.28 & 0.36 & 1.11 & $(0.64,1.92)$ & 0.722 \\
\hline No & - & - & - & - & - & \\
\hline \multicolumn{7}{|l|}{ Chemotherapy } \\
\hline Yes & 0.13 & 0.24 & 0.54 & 1.13 & $(0.71,1.80)$ & 0.591 \\
\hline No & - & - & - & - & - & \\
\hline \multicolumn{7}{|l|}{ Hormonal therapy } \\
\hline Yes & -0.01 & 0.28 & -0.05 & 0.99 & $(0.57,1.71)$ & 0.959 \\
\hline No & - & - & - & - & - & \\
\hline Age (in years) & -0.02 & 0.01 & -2.10 & 0.98 & $(0.96,1.00)$ & 0.036 \\
\hline $\begin{array}{l}\text { Years elapsed since the breast } \\
\text { cancer treatment }\end{array}$ & -0.04 & 0.02 & -1.85 & 0.96 & $(0.91,1.00)$ & 0.064 \\
\hline $\begin{array}{l}\text { Numbers of lymph nodes } \\
\text { removed }\end{array}$ & 0.004 & 0.02 & 0.26 & 1.00 & $(0.97,1.04)$ & 0.793 \\
\hline Body fat percentage & 0.01 & 0.02 & 0.57 & 1.01 & $(0.98,1.04)$ & 0.570 \\
\hline Intercept & -1.73 & 0.67 & -2.59 & 0.18 & $(0.09,0.60)$ & 0.010 \\
\hline McFadden's Pseudo $R^{2}$ : & & & & 0.060 & & \\
\hline $\mathrm{LR} \chi^{2}(18)$ & & & & 38.35 & & \\
\hline Prob $>\chi^{2}$ & & & & 0.004 & & \\
\hline
\end{tabular}

${ }^{1}$ B: Regression Coefficient; SE: Standard Error; CI: Confidence Interval; OR: Odds Ratio; -: Reference Group.

\section{Discussion}

This is the first study in a larger sample of breast cancer patients that provides initial evidence for quantifiable effects of obesity defined as a BMI $\geq 30 \mathrm{~kg} / \mathrm{m}^{2}$ on patient-centered health outcomes of lymphatic pain, arm swelling, and truncal swelling. This study extends the previous work that obesity defined as a BMI $\geq 30 \mathrm{~kg} / \mathrm{m}^{2}$ is a risk factor for objective arm swelling or lymphedema defined as an increased limb size or girth comparing affected (or lymphedematous) and unaffected limbs $[19,20]$ as well as lymphedema defined by >7.1 L-Dex ratio by a Bioelectrical Impedance Analysis (BIA) device [28]. Obesity can influence breast cancer patients' inflammatory responses, resulting in abnormal lymph fluid accumulation $[17,18]$. We found that patients with obesity suffered the greatest odds 
of having lymphatic pain compared to clinical risk factors of having radiotherapy and more lymph nodes removed and demographic risk factor of having financial hardship. Similarly, patients with obesity had the highest odds of having arm swelling compared to clinical factors of having a mastectomy, axillary lymph node dissection, radiotherapy and more lymph nodes removed as well as demographic factor of having financial hardship. Thus, the results support our study hypothesis that breast cancer patients with obesity (BMI $\geq 30 \mathrm{~kg} / \mathrm{m}^{2}$ ) would have higher odds of having lymphatic pain and arm swelling than those with nonobesity (BMI $<30 \mathrm{~kg} / \mathrm{m}^{2}$ ) controlling for demographic and clinical characteristics as well as body fat percentage. Obesity had no effects on truncal swelling. Furthermore, having a mastectomy and younger age had very small effects on truncal swelling. This suggests that truncal swelling may have different pathological underpinnings. As obesity, lymphatic pain, and arm swelling are inflammatory pathological conditions $[4,17,18]$, future research should explore the inflammatory pathways and understand the molecular mechanisms to find a cure for lymphatic pain and swelling, even for lymphedema.

About one in four patients in our study were obese (BMI $\left.\geq 30 \mathrm{~kg} / \mathrm{m}^{2}\right)$. Significantly higher body fat percentage and body fat mass were found in patients with obesity compared to patients with non-obesity. Researchers have speculated that body fat percentage and body fat mass may be more accurate measures for obesity than obesity defined as a BMI $\geq 30 \mathrm{~kg} / \mathrm{m}^{2}$ due to inclusion of lean muscle in BMI [2]. Our results suggest that after controlling for body fat percentage, obesity defined as BMI $\geq 30 \mathrm{~kg} / \mathrm{m}^{2}$ still had the greatest effects on lymphatic pain and arm swelling. Future research should focus on clinical cutoff points for obesity in terms of body fat percentage or body fat mass in breast cancer patients and compare predictive sensitivity among BMI, body fat percentage, and body fat mass on patient-centered health outcomes.

It should be noted that clinical risk factors (i.e., radiotherapy, mastectomy, more lymph nodes removed, axillary lymph node dissection) and demographic risk factor (i.e., younger age) are non-modifiable risk factors. As the major risk factor for lymphatic pain and arm swelling, obesity is the only risk factor that can be modified through lifestyle changes. This underscores the importance of incorporating obesity management in treating and reducing the risk of lymphatic pain and arm swelling.

Importantly, findings of our study also provide new evidence that patients with financial hardship also had higher odds of having lymphatic pain and arm swelling comparing to the aforementioned non-modifiable clinical and demographic risk factors. In our study, patients with obesity were more likely to be non-white, have diabetes, have lymphedema, and have financial hardship. The finding that having financial hardship results in the increased odds of having lymphatic pain and arm swelling in breast cancer patients is noteworthy. Obesity and financial hardship are strongly intertwined with socioeconomic status, and the interconnection of race and socioeconomic status accounts for substantial racial/ethnic differences in health $[29,30]$. Taken together, our study suggests that management of obesity and provision of financial support may be beneficial in reducing the risk of developing long-term and debilitating adverse effects of cancer treatment (e.g., lymphatic pain, lymphedema, swelling) and improve the quality of breast cancer survival and decrease years lost due to disability.

\section{Limitations and Strengths of the study}

Limitations of the study include its cross-sectional design that prevents an evaluation of changes in obesity in relation to lymphatic pain, arm swelling, and truncal swelling over time. Nevertheless, this is the first study that provides initial evidence for quantifiable effects of obesity on patient-centered health outcomes of lymphatic pain, arm swelling, and truncal swelling. A strength of the study is the use of a valid and reliable instrument to evaluate symptoms associated with lymph fluid accumulation, which allows for precision classification of lymphatic pain, arm swelling, and truncal swelling. Another strength of the study is to investigate the effects of obesity (BMI $\geq 30 \mathrm{~kg} / \mathrm{m}^{2}$ ) after controlling for body fat in relation 
to muscle mass (i.e., body fat percentage). Finally, a comparatively larger sample permits to quantify the effects of obesity on lymphatic pain, arm swelling, and truncal swelling.

\section{Conclusions}

Obesity, lymphatic pain, arm swelling, and truncal swelling affect a substantial proportion of breast cancer patients. Findings of our study demonstrate that obesity is a major significant risk for lymphatic pain and arm swelling, even after controlling for the effects of demographic and clinical risk factors as well as body fat percentage. As obesity, lymphatic pain, and swelling are inflammatory pathological conditions $[4,17,18]$, future research should explore the inflammatory pathways and understand the molecular mechanisms to find a cure for lymphatic pain and swelling, and even for lymphedema. In addition, obesity is the only risk factor that can be modified through lifestyle changes while financial hardship may be addressed by societal intervention. A holistic approach that incorporates medical, lifestyle, and social support should be considered in treating and reducing the risk of lymphatic pain and arm swelling.

Author Contributions: Conceptualization, M.R.F., Y.W. and D.A.; methodology, M.R.F., Y.W., D.A.; software, M.L.M. and Z.Z.; validation, M.R.F., M.L.M. and Z.Z.; formal analysis, M.L.M. and Z.Z.; investigation, M.R.F., Y.W., D.A., A.G. and E.K.; resources, M.R.F., D.A., A.G. and J.S.; data curation, M.R.F., D.A., A.G. and E.K.; writing-original draft preparation, M.R.F., J.M.Q., M.L.M., D.A. and Z.Z.; writing-review and editing, M.R.F., J.M.Q., M.L.M., D.A., Z.Z., A.G., J.S., Y.W., C.M.-N. and E.K.; visualization, E.K.; supervision, M.R.F. and Y.W.; project administration, M.R.F., Y.W., D.A., A.G. and J.S.; funding acquisition, M.R.F. and Y.W. All authors have read and agreed to the published version of the manuscript.

Funding: This study was supported by the National Institute of Health/National Science Foundation/National Cancer Institute (1R01CA214085-01) with M.R.F. and Y.W. as the multiple principal investigators. This study was also supported by a research grant from Judges and Lawyers for Breast Cancer Alert with M.R.F. as the principal investigator. Its contents are solely the responsibility of the authors and do not necessarily represent the official views of the funders. The funders had no role in the study design, data collection and analysis, decision to publish, or preparation of the manuscript.

Institutional Review Board Statement: The study (IRB \# 16-01665) was conducted according to the guidelines of the Declaration of Helsinki, and approved by the Institutional Review Board of NYU Langone Health in New York City, the United States. This study did not contain any animal subjects.

Informed Consent Statement: Informed consent was obtained from all subjects involved in the study.

Acknowledgments: We thank Alejandra Yancey for helping manage the study and data collection. We thank all the patients who participated in the study. We thank the nurses, physicians, staff at the NYU Perlmutter Cancer Center for their support for the study.

Conflicts of Interest: The authors declare no conflict of interest.

\section{References}

1. World Health Organization. Obesity and Overweight Fact Sheet. Available online: https://www.who.int/news-room/factsheets/detail/obesity-and-overweight (accessed on 9 May 2021).

2. Hales, C.M.; Carroll, M.D.; Fryar, C.D.; Ogden, C.L. Prevalence of obesity and severe obesity among adults: United States, 2017-2018. NCHS Data Briefs 2020, 360, 1-8.

3. Snitker, S. Use of body fatness cutoff points. Mayo Clin. Proc. 2010, 85, 1057-1058. [CrossRef]

4. Imayama, I.; Alfano, C.M.; Neuhouser, M.L.; George, S.M.; Wilder Smith, A.; Baumgartner, R.N.; Baumgartner, K.B.; Bernstein, L.; Wang, C.Y.; Duggan, C.; et al. Weight, inflammation, cancer-related symptoms and health related quality of life among breast cancer survivors. Breast Cancer Res. Treat. 2013, 140, 159-176. [CrossRef] [PubMed]

5. Agurs-Collins, T.; Ross, S.A.; Dunn, B.K. The many faces of obesity and its influence on breast cancer risk. Front. Oncol. 2019, 9, 765. [CrossRef] [PubMed]

6. Argolo, D.F.; Hudis, C.A.; Iyengar, N.M. The impact of obesity on breast cancer. Curr. Oncol. Rep. 2018, 20, 47. [CrossRef]

7. Chan, D.; Vieira, A.R.; Aune, D.; Bandera, E.V.; Greenwood, D.C.; McTiernan, A.; Navarro Rosenblatt, D.; Thune, I.; Vieira, R.; Norat, T. Body mass index and survival in women with breast cancer-systematic literature review and meta-analysis of 82 follow-up studies. Ann. Oncol. 2014, 25, 1901-1914. [CrossRef] 
8. Brorson, H.; Ohlin, K.; Olsson, G.; Nilsson, M. Adipose tissue dominates chronic arm lymphedema following breast cancer: An analysis using volume rendered CT images. Lymphat. Res. Biol. 2006, 4, 199-210. [CrossRef]

9. Ding, Y.Y.; Yao, P.; Wu, L.; Han, Z.K.; Hong, T.; Zhu, Y.Q.; Li, H.X. Body mass index and persistent pain after breast cancer surgery: Findings from the women's healthy eating and living study and a meta-analysis. Oncotarget 2017, 8, 43332-43343. [CrossRef]

10. Degnim, A.C.; Miller, J.; Hoskin, T.L.; Boughey, J.C.; Loprinzi, M.; Thomsen, K.; Maloney, S.; Baddour, L.M.; Cheville, A.L. A prospective study of breast lymphedema: Frequency, symptoms, and quality of life. Breast Cancer Res. Treat. 2012, 134, 915-922. [CrossRef]

11. Eaton, L.H.; Narkthong, N.; Hulett, J.M. Psychosocial Issues Associated with Breast Cancer-Related Lymphedema: A Literature Review. Curr. Breast Cancer Rep. 2020, 1-9. [CrossRef]

12. World Health Organization. Breast Cancer. Available online: https://www.who.int/news-room/fact-sheets/detail/breast-cancer (accessed on 9 May 2021).

13. American Cancer Society. Breast Cancer Facts \& Figures 2019-2020. Available online: https://www.cancer.org/content/dam/ cancer-org/research/cancer-facts-and-statistics/breast-cancer-facts-and-figures/breast-cancer-facts-and-figures-2019-2020. pdf (accessed on 5 May 2021).

14. Washington, A.E.; Lipstein, S.H. The patient-centered outcomes research institute-promoting better information, decisions, and health. N. Engl. J. Med. 2011, 365, e31. [CrossRef] [PubMed]

15. Fu, M.R.; McTernan, M.L.; Qiu, J.M. The Effects of Kinect-Enhanced Lymphatic Exercise Intervention on Lymphatic Pain, Swelling, and Lymph Fluid Level. Integr. Cancer Ther. 2021, 1-14. [CrossRef]

16. Armer, J.A.; Ostby, P.; Ginex, P.; Beck, M.; Deng, J.; Fu, M.R.; Lasinski, B.B.; Lockwood, S.; Poage, E.; White, J.; et al. ONS Guidelines for Cancer treatment-related lymphedema. Oncol. Nurs. Forum 2020, 47, 518-538. [CrossRef]

17. Fu, M.R.; Aouizerat, B.E.; Yu, G.; Conley, Y.; Axelrod, D.; Guth, A.A.; Gagner, J.-P.; Qiu, J.M.; Zagzag, D. Model-based patterns of lymphedema symptomology: Phenotypic and biomarker characterization. Curr. Breast Cancer Rep. 2020. [CrossRef]

18. Fu, M.R.; Conley, Y.P.; Axelrod, D.; Guth, A.; Yu, G.; Fletcher, J.; Zagzag, D. Precision assessment of heterogeneity of lymphedema phenotype, genotypes and risk prediction. Breast 2016, 29, 231-240. [CrossRef] [PubMed]

19. Fu, M.R.; Axelrod, D.; Cleland, C.M.; Qiu, Z.; Guth, A.A.; Kleinman, R.; Scagliola, J.; Haber, J. Symptom reporting in detecting breast cancer-related lymphedema. Breast Cancer 2015, 7, 345-352. [CrossRef] [PubMed]

20. Helyer, L.K.; Varnic, M.; Le, L.W.; Leong, W.; McCready, D. Obesity is a risk factor for developing postoperative lymphedema in breast cancer patients. Breast J. 2010, 16, 48-54. [CrossRef]

21. Greene, A.K.; Zurakowski, D.; Goss, J.A. Body mass index and lymphedema morbidity: Comparison of obese versus normalweight patients. Plast. Reconstr. Surg. 2020, 146, 402-407. [CrossRef]

22. Wu, R.; Huang, X.; Dong, X.; Zhang, H.; Zhuang, L. Obese patients have higher risk of breast cancer-related lymphedema than overweight patients after breast cancer: A meta-analysis. Ann. Transl. Med. 2019, 7, 172. [CrossRef]

23. Forsythe, L.P.; Alfano, C.M.; George, S.M.; McTiernan, A.; Baumgartner, K.B.; Bernstein, L.; Ballard-Barbash, R. Pain in long-term breast cancer survivors: The role of body mass index, physical activity, and sedentary behavior. Breast Cancer Res. Treat. 2013, 137, 617-630. [CrossRef]

24. Leysen, L.; Beckwée, D.; Nijs, J.; Pas, R.; Bilterys, T.; Vermeir, S.; Adriaenssens, N. Risk factors of pain in breast cancer survivors: A systematic review and meta-analysis. Support Care Cancer 2017, 25, 3607-3643. [CrossRef]

25. Fu, M.R.; Axelrod, D.; Guth, A.A.; Rampertaap, K.; El-Shammaa, N.; Hiotis, K.; Scagliola, J.; Yu, G.; Wang, Y. mHealth self-care interventions: Managing symptoms following breast cancer treatment. mHealth 2016, 2, 28. [CrossRef]

26. Shi, S.; Lu, Q.; Fu, M.R.; Ouyang, Q.; Liu, C.; Lv, J.; Wang, Y. Psychometric properties of the breast cancer and lymphedema symptom experience index: The Chinese version. Eur. J. Oncol. Nurs. 2016, 20, 10-16. [CrossRef]

27. Norton, E.C.; Dowd, B.E.; Maciejewski, M.L. Odds Ratios-Current Best Practice and Use. JAMA 2018, 320, 84-85. [CrossRef] [PubMed]

28. Fu, M.R.; Axelrod, D.; Guth, A.A.; Fletcher, J.; Qiu, J.M.; Scagliola, J.; Kleinman, R.; Ryan CEChan, N.; Haber, J. Patterns of obesity and lymph fluid level during the first year of breast cancer treatment: A prospective study. J. Pers. Med. 2015, 5, 326-340. [CrossRef] [PubMed]

29. Williams, D.R.; Lawrence, J.A.; Davis, B.A. Racism and Health: Evidence and Needed Research. Annu. Rev. Public Health 2019, 40, 105-125. [CrossRef] [PubMed]

30. Naito, R.; Leong, D.P.; Bankdiwala, S.I.; Mckee, M.; Subramanian, S.V.; McKee, M.; Subramanian, S.V.; Rangarajan, S.; Islam, S.; Avezum, A.; et al. Impact if social on mortality and morbidity in 20 high-income, middle-income and low-income countries in five continents. BMJ Glob. Health 2021, 6, e004124. [CrossRef] 